\title{
Production of Metallic Copper Powder by Autocatalytic Reaction in Suspension
}

\author{
João Guilherme Rocha Poço ${ }^{\mathrm{a}}$, Roberto Guardani ${ }^{\mathrm{b} *}$, Cláudia Satie Shimmi $^{\mathrm{a}, \mathrm{b}}$, Marco Giulietti $^{\mathrm{a}, \mathrm{c}}$ \\ ${ }^{a}$ Institute for Technological Research - IPT, Chemistry Division, São Paulo - SP, Brazil \\ ${ }^{\mathrm{b}}$ Chemical Engineering Department, University of São Paulo, \\ Av. Luciano Gualberto, 380, Tv. 3, 05508-900 São Paulo - SP, Brazil \\ ${ }^{c}$ Chemical Engineering Department, Federal University of São Carlos, São Carlos - SP, Brazil
}

Received: April 29, 2004; Revised: December 29, 2005

\begin{abstract}
The production of metallic powder by precipitation from solution was studied in laboratory scale as an alternative to the conventionally adopted processes, based on the atomization of molten material, for producing metal powders with small particle size. The process is based on the precipitation of metals from aqueous solutions by reduction under controlled conditions. Results of laboratory scale experiments are presented for the production of copper particles from aqueous solutions of copper sulfate, using formaldehyde as reducing reactant, and EDTA as complexing agent. The effect of the presence of nuclei was studied. Metallic particles with average sizes in the range from about $0.3 \mu \mathrm{m}$ to about $15 \mu \mathrm{m}$ were obtained. In the process, large particles are formed mainly by aggregation of submicrometric particles, indicating that the particle size distribution of the product depends on the control of particle agglomeration rate.
\end{abstract}

Keywords: metal powder production, precipitation, metallic particles

\section{Introduction}

In recent years there has been a growing interest in the use of metal powders with increasingly smaller particle sizes in the production of metal pieces by powder metallurgy techniques, since smaller particles can result in improved quality of molded pieces. In order to meet specifications of molding industries, it is necessary to develop metal powder production techniques that are able not only to produce smaller particles, but also to control the particle size.

The production of metal powders for injection molding is normally based on atomization techniques, and considerable effort has been dedicated to the development of atomization nozzles and to the study of the effect of melt properties, as well as atomization variables, such as pressure and flow rate, on the resulting PSD of the products ${ }^{1}$.

This article presents results of laboratory scale experiments on the production of metallic powder by an alternative process, based on the precipitation from aqueous solutions. The process is based on oxy-reduction reactions, involving a soluble precipitating agent and a salt of the desired metal. The product can be composed of particles of the own metal if the precipitant is a reduction agent, or an insoluble salt of the metal. In the latter case the precipitated particles should be further processed by conventional reduction processes in order to obtain the metal powder. The main advantage of the solution precipitation route is the possibility to produce metallic particles with size varying from less than $1 \mu \mathrm{m}$ to about $20 \mu \mathrm{m}$. Particles with size within such range are difficult to obtain by conventional atomization techniques.

In recent years, processes based on the use of reducing agents such as hydrazine, aldehydes, or glycols have been operated in commercial scale, mainly by electroless metal plating industries, in the production of printed circuit boards ${ }^{2-4}$. The overall reaction of copper reduction is the result of a series of intermediate stages, and can be represented as:

$$
\begin{aligned}
& \mathrm{CuEDTA}_{2}^{-}+2 \mathrm{HCHO}+4 \mathrm{OH}^{-} \rightarrow \\
& \mathrm{Cu}+\mathrm{H}_{2}+2 \mathrm{H}_{2} \mathrm{O}+2 \mathrm{HCOO}^{-}+\mathrm{EDTA}_{4}^{-}
\end{aligned}
$$

A number of studies have been published concerning the mechanism and kinetics of reaction 1 , due to its importance in the production of printed circuit boards by electroless copper deposition $^{5-9}$. A discussion on the mechanism of the autocatalytic copper deposition, as well as on parallel reactions that may take place in the system was presented by van den Meerakker and Scholtens ${ }^{5}$, and by Schumacher et al. ${ }^{6}$ According to these authors the main parallel reactions that affect the process are those leading to the decomposition of formaldehyde, like the Cannizzaro reaction, represented by reaction 2:

$$
2 \mathrm{HCHO}+\mathrm{OH}^{-} \leftrightarrow \mathrm{H}_{3} \mathrm{C}-\mathrm{OH}+\mathrm{HCOO}^{-}
$$

Besides these reactions others can lead to the formation of oxalate. Given the complexity of the reaction mechanism, and the difficulty in measuring concentrations of unstable intermediates, no phenomenological model for the reaction rate has been derived, and only empirical models, based on apparent kinetics, have been proposed. This limits the application of the results in systems that operate under conditions different than those studied by the authors ${ }^{5,6}$.

In the present work, metallic copper particles were produced by means of an autocatalytic deposition reaction, in a suspension consisting of colloidal particles (nuclei) of silver, palladium, or copper. The process involves initially the addition of metallic nuclei to a solution of a salt of the desired metal to be produced. The nuclei can be composed of the same metal, but different metals can be used. Alternatively, the metallic nuclei can be formed in the first step of the process, in the vessel containing the solution of the desired metal. For example, when silver particles are added as nuclei to produce copper particles the reaction is initially catalyzed by the silver nuclei, until a 
layer of deposited copper is formed on the colloidal particles. From that point on, the reaction proceeds in an autocatalytic way.

The aim of this work was to identify the main processes that take place, as well as to select adequate conditions in order to control both growth and agglomeration rates of the copper particles.

\section{Experimental}

The following reagents were used in the experiments: sodium hydroxide, copper sulfate $\left(\mathrm{CuSO}_{4} .5 \mathrm{H}_{2} \mathrm{O}\right), \mathrm{Na}_{2}$ EDTA, and formaldehyde (37\% aqueous solution), all as analytical grade. Analytical grade gelatin was added to the solution in experiments in which agglomeration inhibition was tested. Metallic colloidal particles were prepared from solutions of $\mathrm{Ag}_{2} \mathrm{SO}_{4}$ and $\mathrm{PdCl}_{2}$.

The experiments were carried out in a laboratory-scale reactor, which consisted of a jacketed cylindrical glass vessel with $1 \mathrm{~L}$ volume, provided with a mechanical stirrer. The temperature was controlled by means of a thermostatic bath. In all experiments nitrogen gas was continuously injected to the gas volume in the upper part of the reactor, so as to minimize oxidation of the metallic particles. The temperature and the $\mathrm{pH}$ were monitored along the time by thermometers and a $\mathrm{pH}$-meter immersed in the reactor.

The experimental procedure consisted initially of the preparation of $0.034 \mathrm{M}$ copper sulfate and $0.05 \mathrm{M} \mathrm{Na}_{2}$ EDTA aqueous solutions. These concentrations are based on commercially practiced values, as informed in the literature ${ }^{5-9}$, and were kept at the same values in all the experiments. The other components had their concentrations varied according to the values shown in Table 1, which summarizes the conditions adopted in the experiments. Experiments 1 to 5 were carried out discontinuously; experiments 6 to 11 were fed-batch experiments. In all the experiments, after introducing the solution, the reactor was heated up to the desired temperature. The formaldehyde solution was added only after the stability of the temperature, in order to minimize formaldehyde losses by volatilization, or by decomposition reactions. The same reactor was used in fed-batch experiments, in which an extra addition of formaldehyde and $\mathrm{NaOH}(1 \mathrm{M})$ was made by gradually feeding the solutions with the use of a peristaltic pump. In the experiments, both the total mass and the PSD of the metallic particles produced were measured. The measurement of the mass of particles produced in the experiments involved the collection of the suspension at the end of each experiment, followed by filtering under vacuum, drying and weighing.

The PSD of collected samples was measured by forward laser diffraction. This technique is based on the measurement of the angular distribution of the intensity of light diffracted by particles in the path of a light beam. Estimation of the PSD is accomplished by an algorithm based on an optical model for light scattering. A description of the fundamentals of the technique, as well as a discussion on its application in different particle systems can be found elsewhere ${ }^{10,11}$. A Malvern Mastersizer E diffractometer was used in this study. Samples with approximately $10 \mathrm{~mL}$ of suspension were collected from the reactor and dispersed in water in the sample tank of the equipment (approx. $0.5 \mathrm{~L}$ volume), which is equipped with an ultrasound system $(25 \mathrm{kHz})$. All measurements were made at constant obscuration rate (ca. 12\%), within the recommended range by the equipment supplier (from 10 to $30 \%$ ), in order to avoid multiple scattering effects ${ }^{11}$. Assuming $10 \mu \mathrm{m}$ as the volumetric mean particle size, then approximately $10^{11}$ particles were collected from the reactor in each sample. Measurements were made 5 minutes after introduction of each sample in the sample tank of the diffractometer. Then ultrasound was turned on and new measurements were made after 5 minutes.

\section{Results and Discussion}

Preliminary experiments were carried out under condition 1 (Table 1), in order to observe the behavior of the system. The addition of the reducing agent (formaldehyde solution) to the system caused rapid nucleation of silver, resulting in the formation of colloidal particles. As a consequence, an abrupt alteration of the visual aspect of the solution contained in the reactor was observed, from transparent blue to a turbid state and dark color. As the particles had grown by copper deposition a gradual change to brownish color was observed in the suspension. In these experiments the following aspects were observed: 1) the formation of silver or palladium nuclei did not occur at temperatures below about $70{ }^{\circ} \mathrm{C} ; 2$ ) the injection of air in the system apparently caused oxidation of the copper particles (observed

Table 1. Experimental conditions. A) Discontinuous tests.

\begin{tabular}{cccccrr}
\hline Test $\mathrm{n}^{\mathbf{0}}$ & $\mathrm{pH}$ & $\begin{array}{c}\text { Initial HCHO } \\
\text { Concentration }(\mathrm{mL} / \mathrm{L})\end{array}$ & Gelatin $(\mathrm{g} / \mathrm{L})$ & Nature of nuclei ${ }^{1}$; quantity & Temperature $\left({ }^{\circ} \mathrm{C}\right)$ & Time $(\mathrm{min})$ \\
\hline 1 & 12.2 & 4.1 & 0 & $\mathrm{Ag}, \mathrm{R} ; 3.3 \mathrm{mg} / \mathrm{L}$ & 80 & 30 \\
2 & 12.1 & 4.1 & 0.5 & $\mathrm{Ag}, \mathrm{R} ; 2.5 \mathrm{mg} / \mathrm{L}$ & 80 & 80 \\
3 & 12.2 & 4.1 & 0.5 & $\mathrm{Ag}, \mathrm{R} ; \mathrm{Cu} . \mathrm{P}$ & 80 & 140 \\
4 & 12.1 & 4.1 & 0.5 & $\mathrm{Ag}, \mathrm{R} ; 2 \mathrm{mg} / \mathrm{L}$ & 80 & 88 \\
5 & 12.1 & 4.1 & 0.5 & $\mathrm{Pd}, \mathrm{P} ; 0.75 \mathrm{mg} / \mathrm{L}$ & 28 \\
\hline
\end{tabular}

${ }^{1}$ Constituting metal and addition way (R: formed in the reactor; P: pre-formed).

Table 1. Experimental conditions. B) Fed-batch tests.

\begin{tabular}{ccccccccc}
\hline Test $\mathrm{n}^{\circ}$ & $\mathrm{pH}$ & $\begin{array}{c}\text { Initial HCHO } \\
\text { Conc. }(\mathrm{mL} / \mathrm{L})\end{array}$ & $\begin{array}{c}\text { Gelatin } \\
(\mathrm{g} / \mathrm{L})\end{array}$ & $\begin{array}{c}\mathrm{HCHO} \text { feed rate } \\
(\mathrm{mL} / \mathrm{min})\end{array}$ & $\begin{array}{c}\mathrm{NaOH} \text { feed rate } \\
(\mathrm{mL} / \mathrm{min})\end{array}$ & $\begin{array}{c}\text { Nature of nuclei }{ }^{1} ; \\
\text { quantity }\end{array}$ & Temperature $\left({ }^{\circ} \mathrm{C}\right)$ & Time $(\mathrm{min})$ \\
\hline 6 & 12.4 & 4.1 & 0.05 & 0.036 & 0.964 & $\mathrm{Ag}, \mathrm{R} ; 2.5 \mathrm{mg} / \mathrm{L}$ & 80 & 68 \\
7 & 12.4 & 0 & 0.05 & 0.08 & 0 & $\mathrm{Ag}, \mathrm{R} ; 2.5 \mathrm{mg} / \mathrm{L}$ & 80 & 53 \\
8 & 12.6 & 4.1 & 0.05 & 0.08 & 0 & $\mathrm{Ag}, \mathrm{P} ; 0.02 \mathrm{mg} / \mathrm{L}$ & 80 & 70 \\
9 & 12.6 & 8.2 & 0.05 & 0.2 & 0 & $\mathrm{Ag}, \mathrm{P} ; 0.02 \mathrm{mg} / \mathrm{L}$ & 80 & 90 \\
10 & 12.6 & 8.2 & 0.05 & 0.4 & 0.4 & $\mathrm{Ag}, \mathrm{P} ; 0.02 \mathrm{mg} / \mathrm{L}$ & 80 & 90 \\
11 & 12.7 & 8.2 & 0.05 & 0.4 & 0.4 & $\mathrm{Ag}, \mathrm{P} ; 0.02 \mathrm{mg} / \mathrm{L}$ & 80 & 90 \\
\hline
\end{tabular}

${ }^{\mathrm{i}}$ Constituting metal and addition way (R: formed in the reactor; P: pre-formed). 
by the loss of brownish color), thus reverting the process. Air injection should thus be avoided if the objective is to maximize the amount of particles formed; 3 ) the decomposition reactions of formaldehyde were intense in the conditions adopted in the experiments. Therefore the amount of precipitated copper was considerably less than the stoichometrically expected value, based on the total amount of formaldehyde added. The maximum recovered amount of copper was $0.5 \mathrm{~g}$ per experiment, corresponding to about $23 \%$ yield.

The experimental results summarized in Table 2 consist of the dominant size (mode) and the median size of the precipitated copper particles. Since the median value is not affected by extreme values in a distribution, the median size was adopted in the discussion, in order to minimize interference by bubbles, which can affect the PSD measurements at the upper extreme of the size range. The growth of metallic particles was affected by agglomeration, as can be seen in Tables 1 and 2 by comparing Tests 1 and 2 . Addition of gelatin (lyophilic gel), starting from Test 2, prevented particle growth and resulted in particles with median sizes of about $0.3 \mu \mathrm{m}$, which stayed approximately constant along the time. The same effect can be observed in Tests 2, 3, and 4. In Test 3, copper particles produced under the same conditions of Test 2 were used as a source of nuclei, in order to increase the size of the particles produced. The results in Table 2 indicate that the particles did not grow further, and the final product had median size of about $0.3 \mu \mathrm{m}$. Thus, the addition of gelatin has apparently prevented the particles from growing to larger sizes. This observation is in agreement with the literature describing the effect of protective agents like gelatin, which acts as a growth inhibitor and anticoagulant in colloidal systems ${ }^{12,13}$. The presence of protective agents is especially important to avoid agglomeration, even when EDTA is used as a chelating agent, when the concentration of the precipitating species is high. In Test 4 , the formation of agglomerates was observed, resulting in a bimodal PSD curve with dominant sizes equal to 0.37 and $2.8 \mu \mathrm{m}$. The agglomerates could be easily broken by applying ultrasound to the suspension, during measurement of the PSD ( 5 minutes, $25 \mathrm{kHz}$ ). In Test 5 silver nuclei were replaced with palladium. In this case, the nuclei were previously formed by the reaction of a solution of $\mathrm{PdCl}_{2}$ with hydrazine before feeding the reactor.This resulted in an increase of the particle growth rate, with the production of significantly larger particles. By applying ultrasound to the system the median size decreased from 7 to less than $4 \mu \mathrm{m}$, indicating that agglomeration was still influencing the process.

The results of Tests 1 to 5 indicate that addition of gelatin has a significant effect on the tendency of smaller particles to agglomerate. However, growth rate of the particles is also strongly affected, probably due to the growth inhibition effect reported in the literature ${ }^{12,13}$. Tests 6 to 11 were carried out in order to verify favorable conditions to the growth of individual particles to sizes above $1 \mu \mathrm{m}$. In these tests the following conditions were adopted: 1) the gelatin content was lowered to $1 / 10$ of the initially adopted value; 2) formaldehyde was fed in a gradual way, in order to compensate for the effects of decomposition reactions and evaporation; 3 ) in Tests 8 to 11 previously formed silver nuclei were added to the reactor in the beginning of the particle growth step as a way to reduce the number of nuclei in the reactor; 4) in Tests 8 to 11 the total amount of silver nuclei was decreased to $1 / 100$ of the initially adopted value; 5) in Tests 6,10 and 11, a complementary addition of $\mathrm{NaOH}$ was adopted, in order to compensate for the consumption of this reagent during the test.

The results in Table 2 indicate that the particles can grow to sizes larger than $1 \mu \mathrm{m}$ when the metallic nuclei are previously formed, and when formaldehyde is present in the beginning and is added along the test. The addition of smaller amounts of nuclei, in Tests 8 to 11, resulted in monomodal PSD curves with larger particles. In Tests 9, 10 and 11, the initial formaldehyde concentration was larger than in the previous ones and, besides, there was continuous feeding of that reagent. As a consequence, a significant growth of the particles was attained to median sizes of about 6 to $8 \mu \mathrm{m}$. Under such conditions apparently no reaction blocking took place due to the presence of gelatin. In all the tests there was a strong tendency to particle agglomeration, as can be observed from the large difference between median sizes of the products before and after ultrasound. Even in Test 11 , in which particles with median sizes as high as $30 \mu \mathrm{m}$ were produced, the application of ultrasound resulted in the disaggregation to particles with median size of about $6 \mu \mathrm{m}$.

Thus, since no blocking has been observed in Tests 6 to 11, particle agglomeration was the dominant mechanism of particle growth in the process. Since Test 11 resulted in particles with particularly large median size $(30 \mu \mathrm{m})$ it is interesting to observe the evolution of the PSD along the reaction time. This is shown in Figures 1 to 4 . Figure 1 shows a plot of the median size of particles along the reaction time, indicating that up to about $6 \mu \mathrm{m}$ median size the particles are not disaggregated by ultrasound. However, larger particles can be disaggregated by ultrasound, resulting in particles with about $6 \mu \mathrm{m}$ in median size. In Figure 2, the PSD of samples collected at three different times is shown. The effect of agglomeration can be observed in Figure 3, which shows the effect of ultrasound on the PSD of the sample collected at 20 minutes. The PSD shows a second mode at sizes around 0.5 to $0.6 \mu \mathrm{m}$. By applying ultrasound during 5 minutes

Table 2. Experimental results.

\begin{tabular}{ccccc}
\hline Test $^{\circ}$ & Aspect of the PSD & Mode, prior to ultrasound $(\mu \mathrm{m})$ & Median size, prior to ultrasound $(\mu \mathrm{m})$ & Median size, after ultrasound $(\mu \mathrm{m})$ \\
\hline 1 & Monomodal & 5.47 & 5.47 & 3.77 \\
2 & Monomodal & 0.38 & 0.38 & - \\
3 & Monomodal & 0.33 & 0.33 & - \\
4 & Bimodal & $0.37 ; 2.8$ & 0.32 & 3.82 \\
5 & Monomodal & 8.0 & 7.01 & 1.61 \\
6 & Bimodal & $0.27 ; 9.0$ & 7.12 & 0.38 \\
7 & Bimodal & $0.25 ; 5.0$ & 0.39 & 0.49 \\
8 & Monomodal & 2.9 & 2.74 & 1.54 \\
9 & Monomodal & 4.0 & 3.99 & 6.80 \\
10 & Monomodal & 7.89 & 7.89 & 5.78 \\
11 & Monomodal & 30.0 & 30.0 & \\
\hline
\end{tabular}




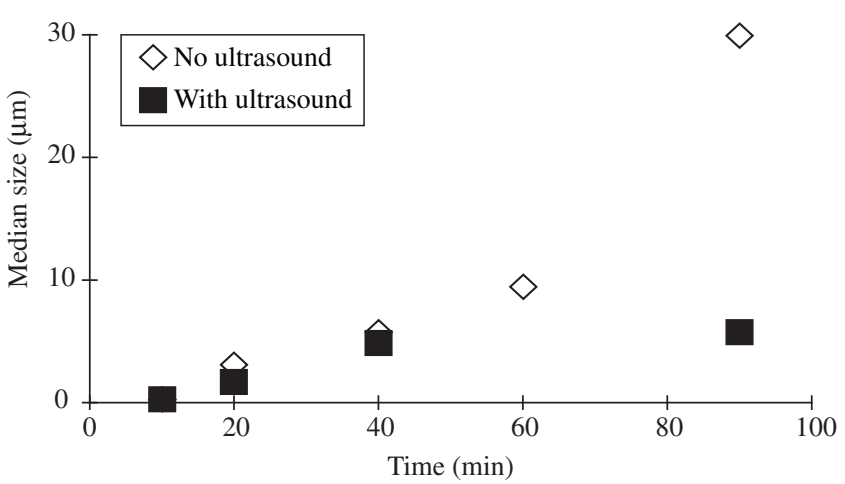

Figure 1. Median size of copper particles produced in Test 11, along the reaction time.

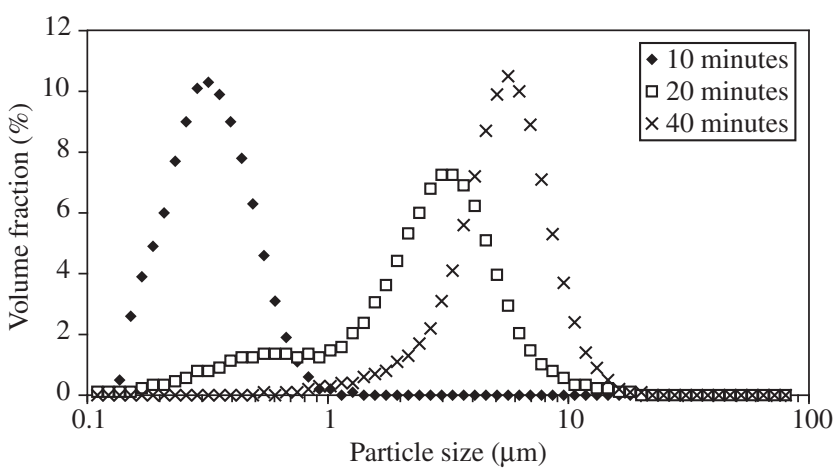

Figure 2. PSD curves of copper particles produced in Test 11, collected at different reaction times.

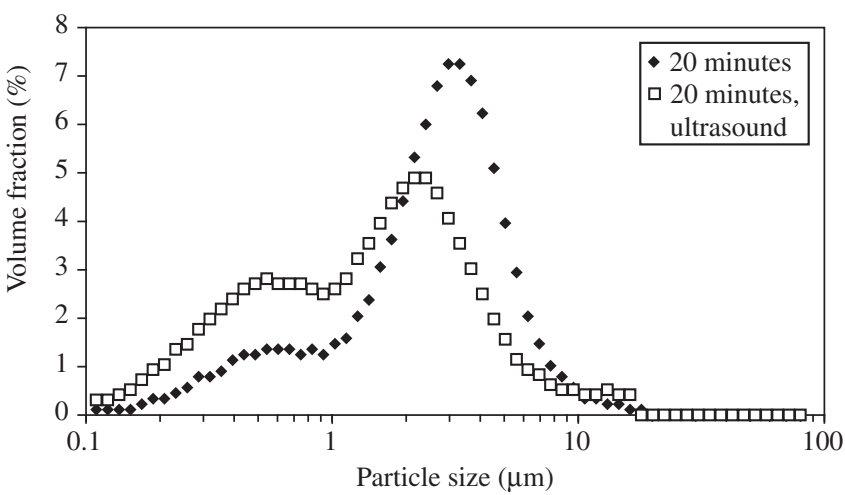

Figure 3. PSD curves of copper particles produced in Test 11, collected after 20 minutes of reaction time, before and after application of ultrasound (5 minutes)

an increase in the height of this second mode in the PSD is observed, as a consequence of disaggregation of the agglomerates. The same procedure was adopted with the sample collected at 40 minutes of reaction (Figure 4). In this case, however, although some increase is observed in smaller sizes (around $0.5 \mu \mathrm{m}$ ), no bimodal curve was obtained, possibly as a consequence of increased cohesion forces of the particles. However, the mode decreased from about 6 to about $4 \mu \mathrm{m}$ after application of ultrasound for 5 minutes.

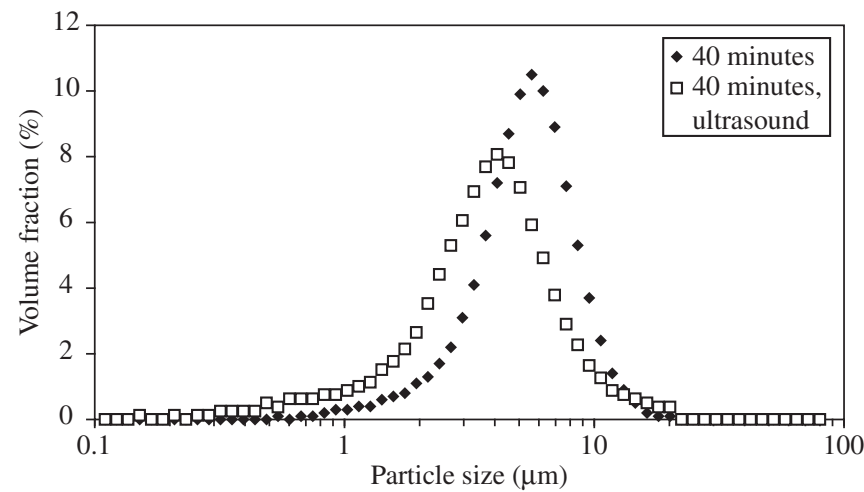

Figure 4. PSD curves of copper particles produced in Test 11, collected after 40 minutes of reaction time, before and after application of ultrasound (5 minutes).

\section{Conclusions}

The results of the laboratory scale experiments indicated that it is possible to produce metallic particles with controllable sizes by reduction in aqueous solution. Since the dominant mechanism affecting particle growth is apparently agglomeration, then the control of the PSD of the product depends mainly on the control of the agglomeration tendency of the particles. This was possible with the use of agglomeration inhibitors, like gelatin. It is necessary, however, to control the concentration of the inhibitor, in order to prevent blocking of the particle surfaces, thus interrupting the growth process.

Addition of previously formed particles that act as nuclei to the further growth of metal particles has resulted in more stable process, as well as products with larger particles. Other important factors in the process are the concentration of formaldehyde and $\mathrm{NaOH}$, since they affect the growth rate of the particles.

Operation under fed-batch regime enables better control of the reagents concentrations along the reaction time, thus contributing to improve the control of process conditions. In the present study, however, no significative effect of the operation regime on the product could be observed, based on the median size of the particles obtained. In order to obtain more information on the process, a systematic study should be carried out to verify the repeatability of the experimental procedure, as well as to quantify the effect of each variable, and the effect of other reagents on the PSD and the properties of the particles produced in the process.

\section{References}

1. Capus JM. Metal Powders: A Global Survey of Production, Applications and Markets. $3^{\text {rd }}$. edition. Amsterdam: Elsevier; 2000.

2. Klar E, editor. Powder Metallurgy, In: Metals Handbook, vol. 7, $9^{\text {th }}$ edition. Metal Park: Ohio; American Society for Metals; 1984.

3. Kaysser WA, Weise W. Powder metallurgy and sintered materials. In: Ullmann's Encyclopedia of Industrial Chemistry, $5^{\text {th }}$ edition. Weinheim: VCH. 1993; A22:105-142.

4. Giulietti M, Danese M, Guardani R. Production of metallic powdered cobalt by precipitation in solution. In: Rojkowski, ZH, editor. Proceedings of the $12^{\text {th }}$ Symposium on Industrial Crystallization; 1993 September 21-23; Warshaw: Poland. Warshaw: Poitechnika Warszawska; 1993. p. $5-157-5-161$.

5. Van Den Meerakker JEAM, Scholtens E. Side reactions in electroless copper solutions with formaldehyde as reducing agent. Berichte der Bunsen-Gesellschaft. 1989; 93(7):786-791.

6. Schumacher R, Pesek KK, Melroy. Kinetic-analysis of electroless deposition of copper. Journal of Physical Chemistry. 1985; 89(20):4338-4342. 
7. Molenaar A, Holdrinet MFE, Van Beek LKH. Kinetics of electroless copper plating with EDTA as the complexing agent for cupric ions. Plating. 1974; 61(3):238-242.

8. El-Raghy SM, Abo-Salama AA. The electrochemistry of electroless deposition of copper. Journal of the Electrochemical Society. 1979; 126(2):171-176.

9. Wiese H, Weil KG. On the mechanism of electroless copper deposition. Berichte der Bunsen-Gesellschaft. 1987; 91(6):619-626.

10. Jones AR. Light scattering for particle characterization. Progress in Energy and Combustion Science. 1999; 25(1):1-53.
11. Guardani R, Nascimento CAO, Onimaru RS. Use of neural networks in the analysis of particle size distribution by laser diffraction: tests with different particle systems. Powder Technology. 2002; 126(1):42-50.

12. Sugimoto T, editor. Fine Particles: Synthesis, Characterization, and Mechanisms of Growth, v. 92, Marcel Dekker: New York; Surfactant Science Series; 2000.

13. Sugimoto T. Monodispersed Particles. Amsterdam; Elsevier, 2001. 